\title{
Chaotic Behaviour in Two-parameter Family of Transcendental Functions Associated with Exponential Map
}

\author{
Mohammad Sajid \\ Mechanical Engineering Department, College of Engineering, \\ Qassim University, Buraidah 51452, Al Qassim, Saudi Arabia. \\ Corresponding author: msajd@qu.edu.sa \\ Abdullah S. Alsuwaiyan \\ Mechanical Engineering Department, Unayzah College of Engineering, \\ Qassim University, Al Qassim, Saudi Arabia. \\ E-mail: asuwaiyan@qec.edu.sa
}

(Received December 21, 2019; Accepted July 15, 2020)

\begin{abstract}
This article is devoted to the study of chaos and bifurcation in the real dynamics of a newly proposed two-parameter family of transcendental functions. We assume that one parameter is continuous and other parameter is discrete. For certain parameters, the theoretical computations of the real fixed points of a family of functions are given. The numerical simulations of the real periodic points of functions are described. The bifurcation diagrams of the real dynamics of these functions for some selected parameter values are provided. In these bifurcation diagrams, the period-doubling occurs which proceeds to a pathway toward chaos in the dynamics of functions. Further, the periodic-three window is visible in the bifurcation diagrams which implies chaos. Lastly, chaos is quantified in the dynamics of functions by calculating Lyapunov exponents.
\end{abstract}

Keywords- Bifurcation, Chaos, Dynamics, Fixed points, Lyapunov exponents, Periodic points.

\section{Introduction}

In many cases, dynamical systems are concerned with mathematical modeling of engineering and scientific systems. With the fast-growing computing tools for both simulation and graphics, various investigations on the real dynamics became more interesting. The real dynamics is to some extent important in studying the dynamics of complex valued functions in the complex plane. It is useful for describing the Fatou sets, the Julia sets and some other related results (Kapoor and Prasad, 1998; Devaney, 2001; Sajid and Kapoor, 2007; Nayak and Prasad, 2014; Sharifi et al., 2016; Chakra and Nayak, 2019). The logistic map $f(x)=r x(1-x), x \in[0,1], r>0$, is widely studied theoretically, computationally, graphically and numerically to explore its dynamical properties such as periodic points, bifurcation and chaotic behaviour. For logistic-like map $f(x)=r x^{\lambda}(1-x)^{\mu}, x \in[0,1]$ with parameters $r>0, \lambda>0$ and $\mu>0$, the bifurcation and chaos are found by Stavroulaki and Sotiropoulos (2012). For some generalized logistic maps, the real dynamics is described (Radwan, 2013). Often, the dynamics of polynomials and rational functions is somewhat easier than transcendental functions. The chaos in the real dynamics of 1dimensional family of Fibonacci-generating functions is explored (Ozer et al., 2014). For 1parameter families of transcendental functions, the bifurcation and chaos are investigated (de Carvalho and Leonel, 2016; Lim, 2016). The analysis of the real dynamics of iterative methods is reported by Magrenan (2014) involving a one-parameter. The real dynamics of $\left(b^{x}-1\right) / x$ 
International Journal of Mathematical, Engineering and Management Sciences

Vol. 5, No. 6, 1191-1209, 2020

https://doi.org/10.33889/IJMEMS.2020.5.6.090

associated with one-parameter is explained (Sajid, 2016). For two-parameter families of special type of generating functions, the bifurcation and chaos are investigated (Sajid, 2018, 2019). For certain families involving one-parameter, the real fixed points are found by Sajid (2015a, 2015b), Lim (2016). From these research works, it reveals that the fixed points and periodic points play a special role in deciding the dynamics of functions. For this purpose, the periodic points of transcendental functions are investigated in this paper. Bifurcation and chaotic behaviour are also investigated. This research work is expected to be helpful in understanding the dynamics of transcendental functions and in developing new kinds of results.

Some applications of chaotic systems are mentioned by Thompson (2016), Liu and Miao (2018), Letellier (2019). Moreover, chaotic behaviors and their applications are widely observed in numerous fields, such as biology, chemistry, computer science, ecology, economics, environmental science, meteorology, physics and almost all engineering disciplines (Banerjee et al., 2013; Skiadas and Skiadas, 2016). Some more recent applications of the present kind of findings can be seen for one-dimensional map (Agliari et al., 2017; Belyaev and Ryazanova, 2019).

In many cases, this kind of discrete systems become more important when, for the ODE systems, some complex models were transformed into discrete models, namely the Poincaré map. Such Poincaré map, as a discrete system, is an important tool to analyze the dynamics of the continuoustime systems. For such types of applications, we can see (i) walking dynamics of the passive compass-gait model under OGY-based state-feedback control: Emergence of bifurcations and chaos (Gritli and Belghith, 2017), (ii) calculation of the Lyapunov exponents in the compass-gait model under OGY control via a hybrid Poincaré map (Gritli and Belghith, 2015).

In this work, one-dimensional map is given as $x_{n+1}=f\left(x_{n}\right)$, where the point $x_{n}$ belongs to the one-dimensional space of real numbers and $n$ takes only positive integer. For an initial value $x_{0}$, the sequence $\left\{x_{k}\right\}$ can be computed as $x_{1}=f\left(x_{0}\right), x_{2}=f\left(x_{1}\right), x_{3}=f\left(x_{2}\right), \ldots \ldots$ The trajectory $\left\{x_{0}, f\left(x_{0}\right), f^{2}\left(x_{0}\right), \ldots, f^{p-1}\left(x_{0}\right)\right\}$ is called a periodic cycle of period $p$ of periodic point $x_{0}$. If $\left|\left(f^{p}\right)^{\prime}\left(x_{0}\right)\right|<1,\left|\left(f^{p}\right)^{\prime}\left(x_{0}\right)\right|>1$, or $\left|\left(f^{p}\right)^{\prime}\left(x_{0}\right)\right|=1$, the periodic point is called attracting, repelling or neutral (indifferent) respectively. The point $x_{0}$ is called a fixed point of $f(x)$ if $p=1$.

As a parameter varies, qualitative changes in the behaviour of the system can occur at certain parameter values. Each of such a change is known as a bifurcation. In other words, when the parameter crosses through certain value, then the dynamics of the function changes. One method displaying the points, at which a parametrized family of functions bifurcates, is called a bifurcation diagram. The bifurcation diagram of a function is a graph in which the horizontal axis represents values of the parameter and the vertical axis represents higher iterates of the variable. We only use the higher iterates of the variable since the diagram is designed to show eventual behaviour of iterates, such as convergence or periodicity or unpredictability.

The phenomenon of chaos is generally related to the field of dynamical systems and it can be characterized in the dynamics by sensitive dependence on the initial conditions. Appearance of 
International Journal of Mathematical, Engineering and Management Sciences

Vol. 5, No. 6, 1191-1209, 2020

https://doi.org/10.33889/IJMEMS.2020.5.6.090

chaos in systems is combined presence of nonlinear interdependence, order and determinism, and sensitive dependence. The word chaos is derived from the ancient Greek word Xóos, normally it means a state lacking order or predictability. Chaos is a fascinating mathematical and physical phenomenon. The study of chaos shows that simple systems can exhibit complex and unpredictable behaviour (Lakshmanan and Rajaseekar, 2003). Generally, chaos can be defined in many different forms according to conditions, observations or applications of the object. As a notion in the mathematical sense, the chaos is developed about 1980. After that, the following characteristic became common opinion: a deterministic evolution is chaotic if (i) this shows property of sensitivity to initial condition (ii) it consists a nonlinear mechanism confirming the mixing of trajectories and these return back in a bounded domain of the phase space.

The chaos in the dynamics can be identified and quantified by several techniques. It is identified by the period-doubling in bifurcation diagram or observing through nearby time series behaviour. A positive value of the Lyapunov exponent provides chaos in the dynamics of functions (Gulick, 2012; Brown, 2018). For a trajectory $\left\{x_{j}\right\}$, starting from $x_{0}$, the Lyapunov exponent is given by

$$
L=\lim _{j \rightarrow \infty} \frac{1}{j} \sum_{i=0}^{j-1} \ln \left|f^{\prime}\left(x_{i}\right)\right|
$$

The present study is mainly focused on the dynamics of a family of certain transcendental functions. This is a special and new kind of family of nonlinear maps associated to exponential map. Such types of functions are interesting as their dynamics often exhibits chaotic phenomenon. Maps are capable of showing such a behaviour as difference equation. These are easy and fast to simulate on computers where time is discrete (Mickens, 2015). To solve a problem in the dynamical systems, we require to iterate a function in several circumstances. For instance, Newton-Raphson method is utilized to find roots of the equation by an iterative scheme. The following family of transcendental functions depending on two parameters associated with exponential map is considered:

$$
G=\left\{g_{\lambda, \mu}(x)=x+(1-\lambda x) e^{\mu x}: x \in R, \lambda>0, \mu<0\right\} .
$$

We assume here that $\lambda$ is a continuous parameter and $\mu$ is a discrete parameter. This considered family of functions is distinctive from the existing families of functions in previous findings which exhibits chaotic behaviour.

The structure of the present paper is as follows: The real fixed points as well as periodic points of $g_{\lambda, \mu}(x)$ with their nature are first found in Section 2. Bifurcation diagrams of $g_{\lambda, \mu}(x)$ are shown in Section 3 for several parameter values. In Section 4, chaos in the real dynamics is explored by computing Lyapunov exponents. Finally, in Section 5, a short conclusion is provided.

\section{Real Fixed Points and Periodic Points of $g_{\lambda, \mu} \in G$ with their Stability}

For $g_{\lambda, \mu} \in G$, the existence of the real fixed points and their stability are described in the present section. 
International Journal of Mathematical, Engineering and Management Sciences

Vol. 5, No. 6, 1191-1209, 2020

https://doi.org/10.33889/IJMEMS.2020.5.6.090

\subsection{Real Fixed Points of $g_{\lambda, \mu} \in G$}

To investigate the nature of the fixed points, the following lemma is used:

Lemma 2.1 Let $t(x)=1-\frac{e^{\mu x}}{x}, x \in R \backslash\{0\}, \mu<0$. Then, the function $t(x)$ attains local minima at $x=\frac{1}{\mu}$. Its local minimum value is $1-\mu$ and $t\left(x^{*}\right)=-1$, where $x^{*}$ is a solution of the equation $e^{\mu x}-2 x=0$. Moreover, $t(x) \rightarrow+\infty$ as $x \rightarrow-\infty, t(x) \rightarrow+\infty$ as $x \rightarrow 0^{-}$, $t(x) \rightarrow-\infty$ as $x \rightarrow 0^{+}$and $t(x) \rightarrow 1$ as $x \rightarrow+\infty$.

Proof. We have $t^{\prime}(x)=\frac{(1-\mu x) e^{\mu x}}{x^{2}}$. When $t^{\prime}(x)=0$, then $x=\frac{1}{\mu}$.

$$
t^{\prime \prime}(x)=-\frac{\left((\mu x-1)^{2}+1\right) e^{\mu x}}{x^{3}} \quad \text { implies } \quad t^{\prime \prime}(1 / \mu)=-e \mu^{3} .
$$

Hence, $t(x)$ attains local minima at $x=\frac{1}{\mu}$ if $\mu<0$. The local minimum value $t(1 / \mu)$ is given as $1-\mu e$. We can easily see the rest of part by Figure 1.

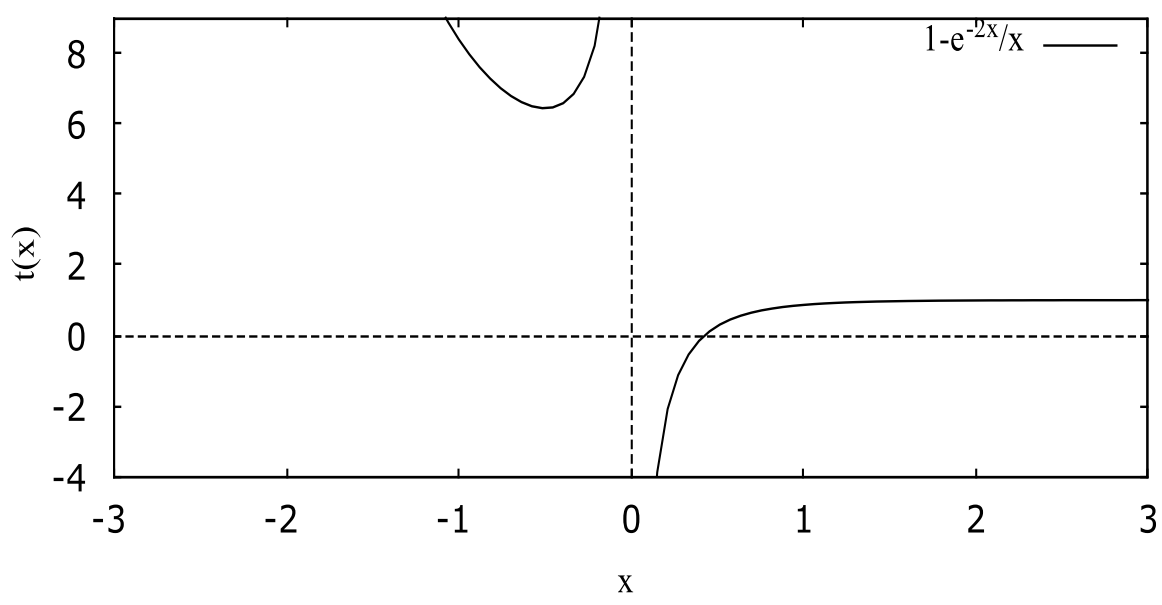

Figure 1. The graph of $t(x)=1-\frac{e^{-2 x}}{x}$

For the family of functions considered, the fixed points of $g_{\lambda, \mu}(x)$ are computed by solving $g_{\lambda, \mu}(x)=x$. It gives us the equation $x+(1-\lambda x) e^{\mu x}=x$. Which provides one fixed point $x_{\lambda}=\frac{1}{\lambda}$, depending on parameter $\lambda$. This fixed point $x_{\lambda}$ does not depend on parameter $\mu$. The nature of $x_{\lambda}$ can be determined as follows 
International Journal of Mathematical, Engineering and Management Sciences

Vol. 5, No. 6, 1191-1209, 2020

https://doi.org/10.33889/IJMEMS.2020.5.6.090

$$
\begin{gathered}
g_{\lambda, \mu}^{\prime}(x)=1+(\mu-\lambda-\mu \lambda x) e^{\mu x} \\
g_{\lambda, \mu}^{\prime}\left(x_{\lambda}\right)=1+\left(\mu-\lambda-\mu \lambda x_{\lambda}\right) e^{\mu x_{\lambda}}
\end{gathered}
$$

Using $\lambda=\frac{1}{x_{\lambda}}$, we have

$$
g_{\lambda, \mu}^{\prime}\left(x_{\lambda}\right)=1-\frac{e^{\mu x_{\lambda}}}{x_{\lambda}} .
$$

(i) For $\mu=-1$, we define $\lambda_{1}=\frac{1}{x_{1}^{*}}(\approx 2.8430599)$, where $x_{1}^{*}(\approx 0.3517337)$ is a solution of the equation $e^{-x}-2 x=0$. We have $g_{\lambda,-1}^{\prime}\left(x_{\lambda}\right)=1-\frac{e^{-x_{\lambda}}}{x_{\lambda}}$. Using Lemma 2.1, we get $-1<g_{\lambda,-1}^{\prime}\left(x_{\lambda}\right)<1$ for $x_{1}^{*}<x_{\lambda}<\infty$. It follows that $\left|g_{\lambda,-1}^{\prime}\left(x_{\lambda}\right)\right|<1$ for $0<\frac{1}{x_{\lambda}}<\frac{1}{x_{1}^{*}}$. Therefore, $g_{\lambda,-1}(x)$ has an attracting fixed point $x_{\lambda}$ for $0<\lambda<\lambda_{1}$. Further, by Lemma 2.1, $g_{\lambda,-1}^{\prime}\left(x_{\lambda}\right)=-1$ for $x_{\lambda}=x_{1}^{*}$. It gives that $\left|g_{\lambda,-1}^{\prime}\left(x_{\lambda}\right)\right|=1$ for $\lambda=\lambda_{1}$. This implies that $g_{\lambda,-1}(x)$ has a rationally indifferent fixed point $x_{\lambda}$ for $\lambda=\lambda_{1}$. Moreover, $g_{\lambda,-1}^{\prime}\left(x_{\lambda}\right)<-1$ for $x_{\lambda}<x_{1}^{*}$ by Lemma 2.1. It shows that $\left|g_{\lambda,-1}^{\prime}\left(x_{\lambda}\right)\right|>1$ for $\lambda>\lambda_{1}$. Thus, the fixed point $x_{\lambda}$ of $g_{\lambda,-1}(x)$ is repelling for $\lambda>\lambda_{1}$.

(ii) For $\mu=-2$, we define $\lambda_{2}=\frac{1}{x_{2}^{*}}(\approx 3.5264462)$, where $x_{2}^{*}(\approx 0.2835716)$ is a solution of the equation $e^{-2 x}-2 x=0$. We have $g_{\lambda,-2}^{\prime}\left(x_{\lambda}\right)=1-\frac{e^{-2 x_{\lambda}}}{x_{\lambda}}$. Using Lemma 2.1, we get $-1<g_{\lambda,-2}^{\prime}\left(x_{\lambda}\right)<1$ for $x_{2}^{*}<x_{\lambda}<\infty$. It provides that $\left|g_{\lambda,-2}^{\prime}\left(x_{\lambda}\right)\right|<1$ for $0<\lambda<\lambda_{2}$. Hence, $g_{\lambda,-2}(x)$ has an attracting fixed point $x_{\lambda}$ for $0<\lambda<\lambda_{2}$. Further, $g_{\lambda,-2}^{\prime}\left(x_{\lambda}\right)=-1$ for $x_{\lambda}=x_{2}^{*}$ by Lemma 2.1. It gives that $\left|g_{\lambda,-2}^{\prime}\left(x_{\lambda}\right)\right|=1$ for $\lambda=\lambda_{2}$. This proves that $g_{\lambda,-2}(x)$ has a rationally indifferent fixed point $x_{\lambda}$ for $\lambda=\lambda_{2}$. Moreover, $g_{\lambda,-2}^{\prime}\left(x_{\lambda}\right)<-1$ for $x_{\lambda}<x_{2}^{*}$ by Lemma 2.1. It shows that $\left|g_{\lambda,-2}^{\prime}\left(x_{\lambda}\right)\right|>1$ for $\lambda>\lambda_{2}$. Thus, $g_{\lambda,-2}(x)$ has a repelling fixed point $x_{\lambda}$ for $\lambda>\lambda_{2}$. 
(iii) For $\mu=-3$, we define $\lambda_{3}=\frac{1}{x_{3}^{*}}(\approx 4.1330216)$, where $x_{3}^{*}(\approx 0.2419537)$ is a solution of the equation $e^{-3 x}-2 x=0$. We have $g_{\lambda,-3}^{\prime}\left(x_{\lambda}\right)=1-\frac{e^{-3 x_{\lambda}}}{x_{\lambda}}$. Applying Lemma 2.1, we have $-1<g_{\lambda,-3}^{\prime}\left(x_{\lambda}\right)<1$ for $x_{3}^{*}<x_{\lambda}<\infty$. It shows that $\left|g_{\lambda,-3}^{\prime}\left(x_{\lambda}\right)\right|<1$ for $0<\lambda<\lambda_{3}$. It provides that $g_{\lambda,-3}(x)$ has an attracting fixed point $x_{\lambda}$ for $0<\lambda<\lambda_{3}$. Further, $g_{\lambda,-3}^{\prime}\left(x_{\lambda}\right)=-1$ for $x_{\lambda}=x_{3}^{*}$ by Lemma 2.1. It gives that $\left|g_{\lambda,-3}^{\prime}\left(x_{\lambda}\right)\right|=1$ for $\lambda=\lambda_{3}$. This implies that $g_{\lambda,-3}(x)$ has a rationally indifferent fixed point $x_{\lambda}$ for $\lambda=\lambda_{3}$. Moreover, $g_{\lambda,-3}^{\prime}\left(x_{\lambda}\right)<-1$ for $x_{\lambda}<x_{3}^{*}$ by Lemma 2.1. It shows that $\left|g_{\lambda,-3}^{\prime}\left(x_{\lambda}\right)\right|>1$ for $\lambda>\lambda_{3}$. This proves that the point $x_{\lambda}$ is a repelling fixed point of $g_{\lambda,-3}(x)$ for $\lambda>\lambda_{3}$.

(iv) For $\mu=-4$, we define $\lambda_{4}=\frac{1}{x_{4}^{*}}(\approx 4.6914998)$, where $x_{4}^{*}(\approx 0.2131515)$ is a solution of the equation $e^{-4 x}-2 x=0$. We have $g_{\lambda,-4}^{\prime}\left(x_{\lambda}\right)=1-\frac{e^{-4 x_{\lambda}}}{x_{\lambda}}$. Applying Lemma 2.1, we have $-1<g_{\lambda,-4}^{\prime}\left(x_{\lambda}\right)<1$ for $x_{4}^{*}<x_{\lambda}<\infty$. Which gives $\left|g_{\lambda,-4}^{\prime}\left(x_{\lambda}\right)\right|<1$ for $0<\lambda<\lambda_{4}$. Consequently, the point $x_{\lambda}$ is an attracting fixed of $g_{\lambda,-4}(x)$ for $0<\lambda<\lambda_{4}$. Further, $g_{\lambda,-4}^{\prime}\left(x_{\lambda}\right)=-1$ for $x_{\lambda}=x_{4}^{*}$ by Lemma 2.1. It gives that $\left|g_{\lambda,-4}^{\prime}\left(x_{\lambda}\right)\right|=1$ for $\lambda=\lambda_{4}$. This proves that $g_{\lambda,-4}(x)$ has a rationally indifferent fixed point $x_{\lambda}$ for $\lambda=\lambda_{4}$. Moreover, $g_{\lambda,-4}^{\prime}\left(x_{\lambda}\right)<-1$ for $x_{\lambda}<x_{4}^{*}$ by Lemma 2.1. It shows that $\left|g_{\lambda,-4}^{\prime}\left(x_{\lambda}\right)\right|>1$ for $\lambda>\lambda_{4}$. Thus, $g_{\lambda,-4}(x)$ possesses a repelling fixed point $x_{\lambda}$ for $\lambda>\lambda_{4}$.

The above cases can be summarized in the following result as:

Proposition 2.1 For each $\mu<0$, there exists a positive real number $\lambda_{\mu}$ such that the unique fixed point $x_{\lambda}=\frac{1}{\lambda}$ of $g_{\lambda, \mu}(x)$ is $(i)$ attracting if $0<\lambda<\lambda_{\mu}$, (ii) rationally indifferent if $\lambda=\lambda_{\mu}$, and (iii) repelling if $\lambda>\lambda_{\mu}$. Here $\lambda_{\mu}=\frac{1}{x_{\mu}^{*}}$, where $x_{\mu}^{*}>0$ is the solution of the equation $e^{\mu x}-2 x=0$.

For various $\mu$, we see that the stability of the fixed points of $g_{\lambda, \mu} \in G$ varies while $\lambda$ passes some values. On crossing $\lambda$ these values, the periodic cycle of period 2 or more come into existence. 
International Journal of Mathematical, Engineering and Management Sciences

Vol. 5, No. 6, 1191-1209, 2020

https://doi.org/10.33889/IJMEMS.2020.5.6.090

\subsection{Simulation of Real Cycles of Period 2, 4, 8, 16 with their Nature}

The theoretical analysis of the periodic cycles of $g_{\lambda, \mu} \in G$ is remarkably complicated. Due to this reason, the simulation of real periodic points of $g_{\lambda, \mu}(x)$ is numerically adopted in the present section. For fixed $\mu$, it is discussed that when parameter $\lambda$ increases beyond certain value, the function $g_{\lambda, \mu}(x)$ has periodic cycle of period 2 or more, i.e., it represents the existence of the periodic cycles of period two, four, eight etc. These periodic points are solutions of $g_{\lambda, \mu}^{k}(x)=g_{\lambda, \mu}^{k-1}(x)+\left(1-\lambda g_{\lambda, \mu}^{k-1}(x)\right) e^{\mu g_{\lambda, \mu}^{k-1}(x)}=x$. In the present work, for $\mu=-1,-2,-3,-4$ , the real periodic cycles of period $2,4,8$ and 16 with their stability are simulated by taking certain values of $\lambda$.

For $\mu=-1$, the cycles of period 2, 4, 8 and 16 of $g_{\lambda, \mu}(x)$ are evaluated by choosing $\lambda=3,3.4$, 3.5 and 3.52 respectively.

* If $\lambda=3$, the period-2 cycle of $g_{\lambda, \mu}(x)$ are obtained as $\tau_{1}=0.60205$ and $\tau_{2}=0.16053$. It follows that

$$
\left|g_{\lambda, \mu}^{\prime}\left(\tau_{1}\right) g_{\lambda, \mu}^{\prime}\left(\tau_{2}\right)\right|=|(-0.20153) \times(-1.99660)| \approx 0.40238<1 .
$$

This proves that $g_{3,-1}(x)$ has an attracting cycle of period-2.

* If $\lambda=3.4$, the period-4 cycle of $g_{\lambda, \mu}(x)$ are calculated as $\tau_{1}=0.94464, \tau_{2}=0.08466$, $\tau_{3}=0.73902$ and $\tau_{4}=0.01660$. It gives that

$$
\begin{aligned}
\left|g_{\lambda, \mu}^{\prime}\left(\tau_{1}\right) g_{\lambda, \mu}^{\prime}\left(\tau_{2}\right) g_{\lambda, \mu}^{\prime}\left(\tau_{3}\right) g_{\lambda, \mu}^{\prime}\left(\tau_{4}\right)\right| & =|0.53799 \times(-2.77838) \times 0.09864 \times(-3.27207)| \\
& \approx 0.48244<1 .
\end{aligned}
$$

It implies that the period- 4 cycle of $g_{3.4,-1}(x)$ is attracting.

* If $\lambda=3.5$, the period- 8 cycle of $g_{\lambda, \mu}(x)$ are determined as $\tau_{1}=1.07191, \tau_{2}=0.12986$, $\tau_{3}=0.60892, \quad \tau_{4}=-0.00639, \quad \tau_{5}=1.02254, \quad \tau_{6}=0.09497, \quad \tau_{7}=0.70210 \quad$ and $\tau_{8}=-0.02008$. It follows that

$$
\begin{gathered}
\left|g_{\lambda, \mu}^{\prime}\left(\tau_{1}\right) g_{\lambda, \mu}^{\prime}\left(\tau_{2}\right) g_{\lambda, \mu}^{\prime}\left(\tau_{3}\right) g_{\lambda, \mu}^{\prime}\left(\tau_{4}\right) g_{\lambda, \mu}^{\prime}\left(\tau_{5}\right) g_{\lambda, \mu}^{\prime}\left(\tau_{6}\right) g_{\lambda, \mu}^{\prime}\left(\tau_{7}\right) g_{\lambda, \mu}^{\prime}\left(\tau_{8}\right)\right|=\mid 0.74381 \\
\times(-2.55283) \times(-0.28847) \times(-3.55138) \times 0.66870 \times(-2.79005) \times(-0.01221) \\
\times(-3.66298) \mid \approx 0.16236<1
\end{gathered}
$$

It provides that the period- 8 cycle of $g_{3.5,-1}(x)$ is attracting. 
International Journal of Mathematical, Engineering and Management Sciences

Vol. 5, No. 6, 1191-1209, 2020

https://doi.org/10.33889/IJMEMS.2020.5.6.090

* If $\lambda=3.52$, the period-16 cycle of $g_{\lambda, \mu}(x)$ are found as $\tau_{1}=0.70038, \tau_{2}=-0.02701$, $\tau_{3}=1.09804, \tau_{4}=0.14246, \tau_{5}=0.57480, \tau_{6}=-0.00113 \tau_{7}=1.00399, \tau_{8}=0.07548$,

$\tau_{9}=0.75642, \quad \tau_{10}=-0.02391, \quad \tau_{11}=1.08649, \quad \tau_{12}=0.13353, \quad \tau_{13}=0.59727$, $\tau_{14}=-0.00939, \tau_{15}=1.03340$ and $\tau_{16}=0.09497$. It gives that

$$
\begin{gathered}
\mid g_{\lambda, \mu}^{\prime}\left(\tau_{1}\right) g_{\lambda, \mu}^{\prime}\left(\tau_{2}\right) g_{\lambda, \mu}^{\prime}\left(\tau_{3}\right) g_{\lambda, \mu}^{\prime}\left(\tau_{4}\right) g_{\lambda, \mu}^{\prime}\left(\tau_{5}\right) g_{\lambda, \mu}^{\prime}\left(\tau_{6}\right) g_{\lambda, \mu}^{\prime}\left(\tau_{7}\right) g_{\lambda, \mu}^{\prime}\left(\tau_{8}\right) g_{\lambda, \mu}^{\prime}\left(\tau_{9}\right) \\
\times g_{\lambda, \mu}^{\prime}\left(\tau_{10}\right) g_{\lambda, \mu}^{\prime}\left(\tau_{11}\right) g_{\lambda, \mu}^{\prime}\left(\tau_{12}\right) g_{\lambda, \mu}^{\prime}\left(\tau_{13}\right) g_{\lambda, \mu}^{\prime}\left(\tau_{14}\right) g_{\lambda, \mu}^{\prime}\left(\tau_{15}\right) g_{\lambda, \mu}^{\prime}\left(\tau_{16}\right)|=|(-0.01994) \\
\times(-3.74142) \times 0.78158 \times(-2.48497) \times(-0.40517) \times(-3.52910) \times 0.63872 \\
\times(-2.94504) \times 0.12823 \times(-3.71558) \times 0.76532 \times(-2.54376) \times(-0.33044) \\
\times(-3.59599) \times 0.68604 \times(-2.80650) \approx 0.82693<1 .
\end{gathered}
$$

Consequently, the period-16 cycle of $g_{3.52,-1}(x)$ is attracting.

For $\mu=-2$, the cycles of period 2, 4, 8 and 16 of $g_{\lambda, \mu}(x)$ are calculated by choosing $\lambda=3.6$, $4.1,4.23$ and 4.243 respectively.

- If $\lambda=3.6$, the period- 2 cycle of $g_{\lambda, \mu}(x)$ are obtained as $u_{1}=0.21500$ and $u_{2}=0.36202$. It follows that

$$
\left|g_{\lambda, \mu}^{\prime}\left(u_{1}\right) g_{\lambda, \mu}^{\prime}\left(u_{2}\right)\right|=|(-1.63590) \times(-0.45119)| \approx 0.73810<1 .
$$

This provides that $g_{3.6,-2}(x)$ has an attracting cycle of period-2.

- If $\lambda=4.1$, the period-4 cycle of $g_{\lambda, \mu}(x)$ are calculated as $u_{1}=0.13338, u_{2}=0.48042$, $u_{3}=0.10943$ and $u_{4}=0.55239$. It gives that

$$
\begin{aligned}
\left|g_{\lambda, \mu}^{\prime}\left(u_{1}\right) g_{\lambda, \mu}^{\prime}\left(u_{2}\right) g_{\lambda, \mu}^{\prime}\left(u_{3}\right) g_{\lambda, \mu}^{\prime}\left(u_{4}\right)\right|= & |(-2.83408) \times 0.17342 \times(-3.17998) \times 0.47974| \\
& \approx 0.74981<1 .
\end{aligned}
$$

It implies that $g_{4.1,-2}(x)$ has an attracting cycle of period-4.

- If $\lambda=4.23$, the period- 8 cycle of $g_{\lambda, \mu}(x)$ are determined as $u_{1}=0.16060, u_{2}=0.39316$, $u_{3}=0.09112, u_{4}=0.60329, u_{5}=0.13893, u_{6}=0.45123, u_{7}=0.08269$ and $u_{8}=0.63381$. It follows that

$$
\begin{gathered}
\left|g_{\lambda, \mu}^{\prime}\left(u_{1}\right) g_{\lambda, \mu}^{\prime}\left(u_{2}\right) g_{\lambda, \mu}^{\prime}\left(u_{3}\right) g_{\lambda, \mu}^{\prime}\left(u_{4}\right) g_{\lambda, \mu}^{\prime}\left(u_{5}\right) g_{\lambda, \mu}^{\prime}\left(u_{6}\right) g_{\lambda, \mu}^{\prime}\left(u_{7}\right) g_{\lambda, \mu}^{\prime}\left(u_{8}\right)\right|=\mid(-2.53302) \\
\times(-0.32276) \times(-3.54962) \times 0.66304 \times(-2.82842) \times 0.02152 \times(-3.68750) \times 0.75567 \mid \\
\approx 0.32629<1 .
\end{gathered}
$$


International Journal of Mathematical, Engineering and Management Sciences

Vol. 5, No. 6, 1191-1209, 2020

https://doi.org/10.33889/IJMEMS.2020.5.6.090

It proves that the period- 8 cycle of $g_{4.23,-2}(x)$ is attracting.

- If $\lambda=4.243$, the period-16 cycle of $g_{\lambda, \mu}(x)$ are found as $u_{1}=0.16055, u_{2}=0.39177$,

$$
\begin{aligned}
& u_{3}=0.08925, u_{4}=0.60899, u_{5}=0.14042, u_{6}=0.44565, u_{7}=0.08027, u_{8}=0.64186, \\
& u_{9}=0.16447, u_{10}=0.38193, u_{11}=0.09285, u_{12}=0.59618, u_{13}=0.13194, u_{14}=0.47001, \\
& u_{15}=0.08164 \text { and } u_{16}=0.63680 . \text { It gives that } \\
& \mid g_{\lambda, \mu}^{\prime}\left(u_{1}\right) g_{\lambda, \mu}^{\prime}\left(u_{2}\right) g_{\lambda, \mu}^{\prime}\left(u_{3}\right) g_{\lambda, \mu}^{\prime}\left(u_{4}\right) g_{\lambda, \mu}^{\prime}\left(u_{5}\right) g_{\lambda, \mu}^{\prime}\left(u_{6}\right) g_{\lambda, \mu}^{\prime}\left(u_{7}\right) g_{\lambda, \mu}^{\prime}\left(u_{8}\right) g_{\lambda, \mu}^{\prime}\left(u_{9}\right) \\
& \times g_{\lambda, \mu}^{\prime}\left(u_{10}\right) g_{\lambda, \mu}^{\prime}\left(u_{11}\right) g_{\lambda, \mu}^{\prime}\left(u_{12}\right) g_{\lambda, \mu}^{\prime}\left(u_{13}\right) g_{\lambda, \mu}^{\prime}\left(u_{14}\right) g_{\lambda, \mu}^{\prime}\left(u_{15}\right) g_{\lambda, \mu}^{\prime}\left(u_{16}\right)|=|(-2.54006) \\
& \times(-0.33310) \times(-3.58886) \times 0.68196 \times(-2.81457) \times(-0.00938) \times(-3.73684) \\
& \times 0.77947 \times(-2.48855) \times(-0.39852) \times(-3.53059) \times 0.64072 \times(-2.93501) \\
& \times 0.11937 \times(-3.71416) \times 0.76518 \mid \approx 0.35572<1 .
\end{aligned}
$$

Consequently, the period-16 cycle of $g_{4.243,-2}(x)$ is attracting.

For $\mu=-3$, the cycles of period 2, 4, 8 and 16 of $g_{\lambda, \mu}(x)$ are computed by selecting $\lambda=4.5$, $4.8,4.875$ and 4.89 respectively.

- If $\lambda=4.5$, the period- 2 cycle of $g_{\lambda, \mu}(x)$ are obtained as $v_{1}=0.35888$ and $v_{2}=0.14934$. It follows that

$$
\left|g_{\lambda, \mu}^{\prime}\left(v_{1}\right) g_{\lambda, \mu}^{\prime}\left(v_{2}\right)\right|=|0.09529 \times(-2.50364)| \approx 0.23857<1 .
$$

This proves that $g_{4.5,-3}(x)$ has an attracting cycle of period-2.

- If $\lambda=4.8$, the period-4 cycle of $g_{\lambda, \mu}(x)$ are found as $v_{1}=0.34323, v_{2}=0.11199$, $v_{3}=0.44246$ and $v_{4}=0.14446$. It gives that

$$
\begin{aligned}
\left|g_{\lambda, \mu}^{\prime}\left(v_{1}\right) g_{\lambda, \mu}^{\prime}\left(v_{2}\right) g_{\lambda, \mu}^{\prime}\left(v_{3}\right) g_{\lambda, \mu}^{\prime}\left(v_{4}\right)\right|= & \mid(-0.02044) \times(-3.42164) \times 0.62118 \\
& \times(-2.70825) \mid \approx 0.11764<1 .
\end{aligned}
$$

It provides that the period-4 cycle of $g_{4.8,-3}(x)$ is attracting.

- If $\lambda=4.875$, the period- 8 cycle of $g_{\lambda, \mu}(x)$ are determined as $v_{1}=0.44996, v_{2}=0.14050$, $v_{3}=0.34719, v_{4}=0.10279, v_{5}=0.46931, v_{6}=0.15423, v_{7}=0.31045$ and $v_{8}=0.10814$. It follows that 
International Journal of Mathematical, Engineering and Management Sciences

Vol. 5, No. 6, 1191-1209, 2020

https://doi.org/10.33889/IJMEMS.2020.5.6.090

$$
\begin{gathered}
\left|g_{\lambda, \mu}^{\prime}\left(v_{1}\right) g_{\lambda, \mu}^{\prime}\left(v_{2}\right) g_{\lambda, \mu}^{\prime}\left(v_{3}\right) g_{\lambda, \mu}^{\prime}\left(v_{4}\right) g_{\lambda, \mu}^{\prime}\left(v_{5}\right) g_{\lambda, \mu}^{\prime}\left(v_{6}\right) g_{\lambda, \mu}^{\prime}\left(v_{7}\right) g_{\lambda, \mu}^{\prime}\left(v_{8}\right)\right|=\mid 0.66440 \\
\times(-2.81832) \times 0.01281 \times(-3.68096) \times 0.75257 \times(-2.53793) \times(-0.31390) \\
\times(-3.54980) \mid \approx 0.18797<1 .
\end{gathered}
$$

It says that $g_{4.875,-3}(x)$ has an attracting cycle of period-8.

- If $\lambda=4.89$, the period-16 cycle of $g_{\lambda, \mu}(x)$ are calculated as $v_{1}=0.45293, v_{2}=0.14075$, $v_{3}=0.34510, v_{4}=0.10094, v_{5}=0.47503, v_{6}=0.15689, v_{7}=0.30229, v_{8}=0.10920$, $v_{9}=0.44504, v_{10}=0.13554, v_{11}=0.36010, v_{12}=0.10178, v_{13}=0.47190, v_{14}=0.15447$ , $v_{15}=0.30838$ and $v_{16}=0.10698$. It gives that

$$
\begin{aligned}
& \mid g_{\lambda, \mu}^{\prime}\left(v_{1}\right) g_{\lambda, \mu}^{\prime}\left(v_{2}\right) g_{\lambda, \mu}^{\prime}\left(v_{3}\right) g_{\lambda, \mu}^{\prime}\left(v_{4}\right) g_{\lambda, \mu}^{\prime}\left(v_{5}\right) g_{\lambda, \mu}^{\prime}\left(v_{6}\right) g_{\lambda, \mu}^{\prime}\left(v_{7}\right) g_{\lambda, \mu}^{\prime}\left(v_{8}\right) g_{\lambda, \mu}^{\prime}\left(v_{9}\right) \\
& \times g_{\lambda, \mu}^{\prime}\left(v_{10}\right) g_{\lambda, \mu}^{\prime}\left(v_{11}\right) g_{\lambda, \mu}^{\prime}\left(v_{12}\right) g_{\lambda, \mu}^{\prime}\left(v_{13}\right) g_{\lambda, \mu}^{\prime}\left(v_{14}\right) g_{\lambda, \mu}^{\prime}\left(v_{15}\right) g_{\lambda, \mu}^{\prime}\left(v_{16}\right)|=| 0.67994 \\
& \times(-2.81872) \times(-0.00407) \times(-3.73466) \times 0.77844 \times(-2.49040) \times(-0.39522) \\
& \quad \times(-3.53150) \times 0.64179 \times(-2.92998) \times 0.11483 \times(-3.71362) \times 0.76519 \\
& \quad \times(-2.53816) \times(-0.33460) \times(-3.58538) \mid \approx 0.14730<1 .
\end{aligned}
$$

Therefore, the period-16 cycle of $g_{4.89,-3}(x)$ is attracting.

For $\mu=-4$, the cycles of period 2, 4, 8 and 16 of $g_{\lambda, \mu}(x)$ are simulated by considering $\lambda=4.8$, 5.4, 5.47 and 5.49 respectively.

- If $\lambda=4.8$, the period- 2 cycle of $g_{\lambda, \mu}(x)$ are obtained as $w_{1}=0.17328$ and $w_{2}=0.25741$. It follows that

$$
\left|g_{\lambda, \mu}^{\prime}\left(w_{1}\right) g_{\lambda, \mu}^{\prime}\left(w_{2}\right)\right|=|(-1.73657) \times(-0.37774)| \approx 0.65597<1 .
$$

This implies that $g_{4.8,-4}(x)$ has an attracting cycle of period-2.

- If $\lambda=5.4$, the period-4 cycle of $g_{\lambda, \mu}(x)$ are found as $w_{1}=0.13783, w_{2}=0.28518$, $w_{3}=0.11261$ and $w_{4}=0.36239$. It follows that

$$
\begin{aligned}
&\left|g_{\lambda, \mu}^{\prime}\left(w_{1}\right) g_{\lambda, \mu}^{\prime}\left(w_{2}\right) g_{\lambda, \mu}^{\prime}\left(w_{3}\right) g_{\lambda, \mu}^{\prime}\left(w_{4}\right)\right|=\mid(-2.70085) \times(-0.03552) \times(-3.44077) \\
& \times 0.63098 \mid \approx 0.20828<1 .
\end{aligned}
$$

Therefore, $g_{5.4,-4}(x)$ has an attracting cycle of period-4. 
- If $\lambda=5.47$, the period- 8 cycle of $g_{\lambda, \mu}(x)$ are determined as $w_{1}=0.10618, w_{2}=0.38030$, $w_{3}=0.14432, \quad w_{4}=0.26254, \quad w_{5}=0.10996, \quad w_{6}=0.36666, \quad w_{7}=0.13466 \quad$ and $w_{8}=0.28836$. It gives that

$$
\begin{gathered}
\left|g_{\lambda, \mu}^{\prime}\left(w_{1}\right) g_{\lambda, \mu}^{\prime}\left(w_{2}\right) g_{\lambda, \mu}^{\prime}\left(w_{3}\right) g_{\lambda, \mu}^{\prime}\left(w_{4}\right) g_{\lambda, \mu}^{\prime}\left(w_{5}\right) g_{\lambda, \mu}^{\prime}\left(w_{6}\right) g_{\lambda, \mu}^{\prime}\left(w_{7}\right) g_{\lambda, \mu}^{\prime}\left(w_{8}\right)\right| \\
=\mid(-3.67352) \times 0.74899 \times(-2.54383) \times(-0.30358) \times(-3.55025) \times 0.66608 \\
\times(-2.80672) \times 0.00267 \mid \approx 0.03759<1 .
\end{gathered}
$$

It proves that the period- 8 cycle of $g_{5.47,-4}(x)$ is attracting.

- If $\lambda=5.49$, the period-16 cycle of $g_{\lambda, \mu}(x)$ are calculated as $w_{1}=0.30016, w_{2}=0.10515$,

$$
\begin{aligned}
& w_{3}=0.38274, \quad w_{4}=0.14451, \quad w_{5}=0.26042, \quad w_{6}=0.10879, \quad w_{7}=0.36942, \\
& w_{8}=0.13484, w_{9}=0.28629, \quad w_{10}=0.10438, \quad w_{11}=0.38560, \quad w_{12}=0.14673, \\
& w_{13}=0.25486, w_{14}=0.11084, w_{15}=0.36214 \text { and } w_{16}=0.13002 . \text { It follows that }
\end{aligned}
$$

$$
\begin{aligned}
& \mid g_{\lambda, \mu}^{\prime}\left(w_{1}\right) g_{\lambda, \mu}^{\prime}\left(w_{2}\right) g_{\lambda, \mu}^{\prime}\left(w_{3}\right) g_{\lambda, \mu}^{\prime}\left(w_{4}\right) g_{\lambda, \mu}^{\prime}\left(w_{5}\right) g_{\lambda, \mu}^{\prime}\left(w_{6}\right) g_{\lambda, \mu}^{\prime}\left(w_{7}\right) g_{\lambda, \mu}^{\prime}\left(w_{8}\right) \\
& \times g_{\lambda, \mu}^{\prime}\left(w_{9}\right) g_{\lambda, \mu}^{\prime}\left(w_{10}\right) g_{\lambda, \mu}^{\prime}\left(w_{11}\right) g_{\lambda, \mu}^{\prime}\left(w_{12}\right) g_{\lambda, \mu}^{\prime}\left(w_{13}\right) g_{\lambda, \mu}^{\prime}\left(w_{14}\right) g_{\lambda, \mu}^{\prime}\left(w_{15}\right) g_{\lambda, \mu}^{\prime}\left(w_{16}\right) \mid \\
& =\mid 0.12752 \times(-3.71545) \times 0.76531 \times(-2.54346) \times(-0.33065) \times(-3.59540) \times 0.68570 \\
& \quad \times(-2.80716) \times(-0.01909) \times(-3.74104) \times 0.78140 \times(-2.48525) \times(-0.40464) \\
& \quad \times(-3.52920) \times 0.63887 \times(-2.94424) \mid \approx 0.78605<1 .
\end{aligned}
$$

Thus, $g_{5.49,-4}(x)$ has an attracting cycle of period-16.

\subsection{Simulation of Real Cycles of Period-3 with their Nature}

For $\mu=-1,-2,-3,-4$, the real cycles of period-3 corresponding to $\lambda=3.9,4.65,5.3$ and 5.92 respectively are determined here with their nature as follows:

- If $\mu=-1$ and $\lambda=3.9$, the period-3 cycle of $g_{\lambda, \mu}(x)$ are given as $p_{1}=-0.16005$, $p_{2}=1.74606$ and $p_{3}=0.73251$. It follows that

$$
\left|g_{\lambda, \mu}^{\prime}\left(p_{1}\right) g_{\lambda, \mu}^{\prime}\left(p_{2}\right) g_{\lambda, \mu}^{\prime}\left(p_{3}\right)\right|=|(-5.48303) \times 1.33315 \times 0.01782| \approx 0.13025<1 .
$$

Therefore, the period-3 cycle of $g_{3.9,-1}(x)$ is attracting.

- If $\mu=-2$ and $\lambda=4.65$, the period-3 cycle of $g_{\lambda, \mu}(x)$ are determined as $q_{1}=0.00609$, $q_{2}=0.96598$ and $q_{3}=0.46015$. It gives that 
International Journal of Mathematical, Engineering and Management Sciences

Vol. 5, No. 6, 1191-1209, 2020

https://doi.org/10.33889/IJMEMS.2020.5.6.090

$$
\left|g_{\lambda, \mu}^{\prime}\left(q_{1}\right) g_{\lambda, \mu}^{\prime}\left(q_{2}\right) g_{\lambda, \mu}^{\prime}\left(q_{3}\right)\right|=|(-5.51344) \times 1.33806 \times 0.05553| \approx 0.40962<1 .
$$

Hence, the period-3 cycle of $g_{4.65,-2}(x)$ is attracting.

- If $\mu=-3$ and $\lambda=5.3$, the period-3 cycle of $g_{\lambda, \mu}(x)$ are calculated as $r_{1}=0.67786$, $r_{2}=0.33856$ and $r_{3}=0.05087$. It follows that

$$
\left|g_{\lambda, \mu}^{\prime}\left(r_{1}\right) g_{\lambda, \mu}^{\prime}\left(r_{2}\right) g_{\lambda, \mu}^{\prime}\left(r_{3}\right)\right|=|1.32428 \times(-0.05635) \times(-5.43075)| \approx 0.40526<1 .
$$

It proves that $g_{5.3,-3}(x)$ has an attracting cycle of period-3.

- If $\mu=-4$ and $\lambda=5.92$, the period-3 cycle of $g_{\lambda, \mu}(x)$ are computed as $s_{1}=0.06524$, $s_{2}=0.53805$ and $s_{3}=0.28406$. It gives that

$$
\left|g_{\lambda, \mu}^{\prime}\left(s_{1}\right) g_{\lambda, \mu}^{\prime}\left(s_{2}\right) g_{\lambda, \mu}^{\prime}\left(s_{3}\right)\right|=|(-5.45150) \times 1.32788 \times(-0.02520)| \approx 0.18243<1 \text {. }
$$

It provides that $g_{5.92,-4}(x)$ has an attracting cycle of period-3.

The existence of periodic cycles with period more than or equal to 2 and period-three window can be visualized by bifurcation diagrams which are given in the next section.

\section{Period-Doubling and Period-three Window in Bifurcation Diagrams}

In the present section, the dynamical behaviour of $g_{\lambda, \mu} \in G$ is graphically visualized by using graphs of bifurcation diagrams. This is familiar that the occurrence of period-doubling in the dynamics of functions implies a route to chaos and, moreover, periodic points of period three implies chaos (Gulick, 2012). It is seen from Section 2.1 that the stability of the real fixed points of $g_{\lambda, \mu}(x)$ varies when $\lambda$ crosses some values. The appearance of periodic points of period two or greater in the real dynamics are exhibited through bifurcation diagrams in Figures 2, 3, 4 and 5 for $\mu=-1,-2,-3,-4$ using $1 \leq \lambda \leq 7,1 \leq \lambda \leq 7.5,1 \leq \lambda \leq 8$ and $1 \leq \lambda \leq 8.5$ respectively.

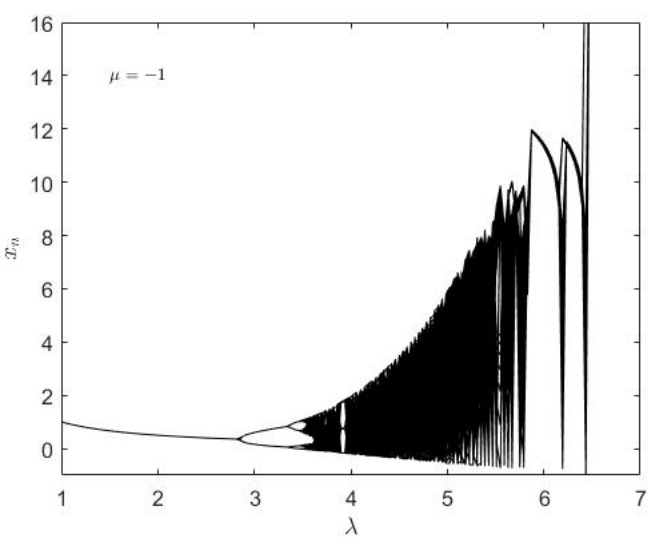

Figure 2. Bifurcation diagram for $\mu=-1$

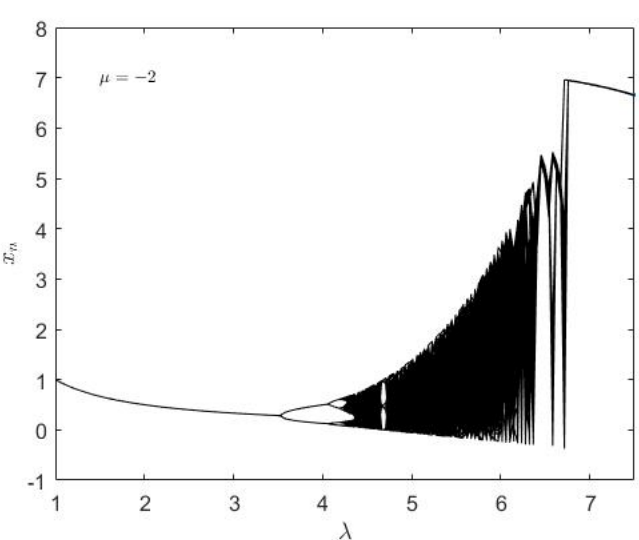

Figure 3. Bifurcation diagram for $\mu=-2$ 
International Journal of Mathematical, Engineering and Management Sciences

Vol. 5, No. 6, 1191-1209, 2020

https://doi.org/10.33889/IJMEMS.2020.5.6.090

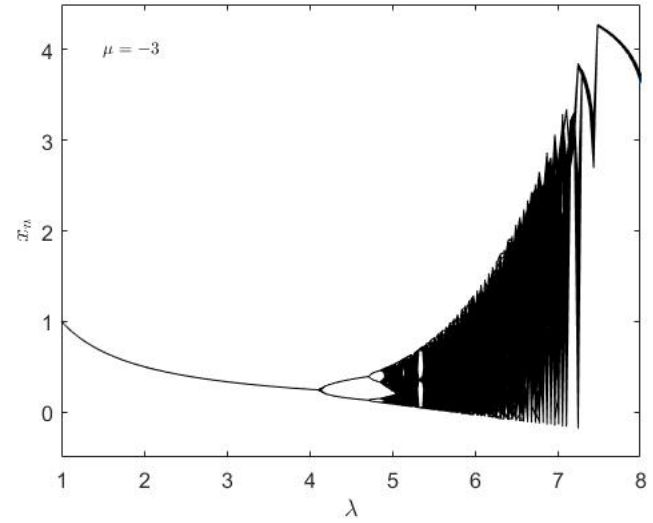

Figure 4. Bifurcation diagram for $\mu=-3$

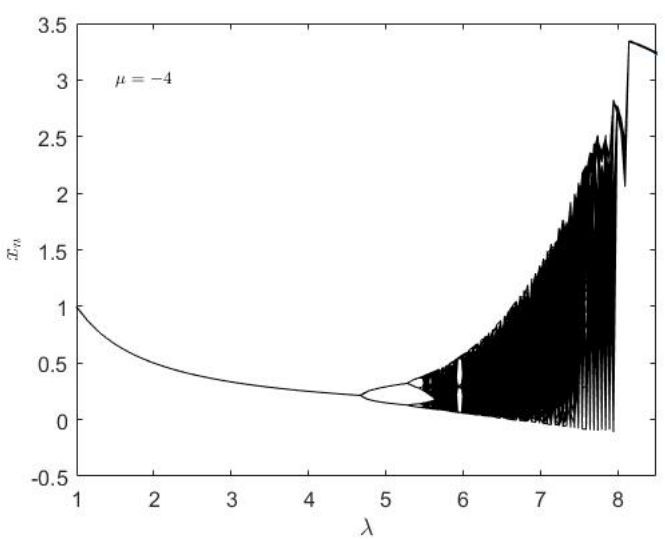

Figure 5. Bifurcation diagram for $\mu=-4$

In these bifurcation diagrams, the period-doubling occurs which proceeds to a pathway toward chaos in the dynamics of $g_{\lambda, \mu} \in G$. When $\mu$ changes from -1 to -4 , then it becomes clear from Figures 2, 3, 4 and 5 that the period-doubling takes place for little larger values of $\lambda$. In these figures, white strips indicate the non-chaotic windows in the bifurcation diagrams.

From the above discussion and numerical calculation, it is evidenced that saddle-node bifurcation happens in the dynamics of $g_{\lambda, \mu} \in G$ which is also demonstrated in Figures 2, 3, 4 and 5.

Moreover, a periodic-three window is clearly visible in the bifurcation diagrams of $g_{\lambda, \mu}(x)$ for $\mu=-1,-2,-3,-4$ corresponding to $\lambda=3.9,4.65,5.3$ and 5.92 respectively (Figures 6, 7, 8 and 9 ) which interprets chaos in the real dynamics. We can observe ahead of this periodic-3 window, a new periodic- 6 cycle appears. Beyond this a more complicated dynamical region is started.

Remark: One may ask that upto what values of $\lambda$ the period-doubling phenomena occur? To answer this question in the absence of an explicit formula, one can be observe from bifurcation diagrams that for $\mu=-1$, the period-doubling happens upto nearly $\lambda=6.5$, for $\mu=-2$, the period-doubling happens upto nearly $\lambda=6.8$, for $\mu=-3$, the period-doubling happens upto nearly $\lambda=7.2$ and for $\mu=-4$, the period-doubling happens upto nearly $\lambda=8.1$. 


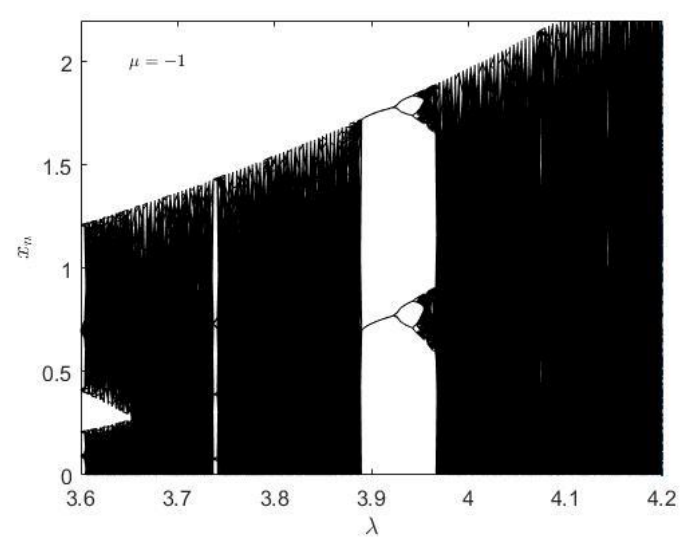

Figure 6. Period-3 window for $\mu=-1$

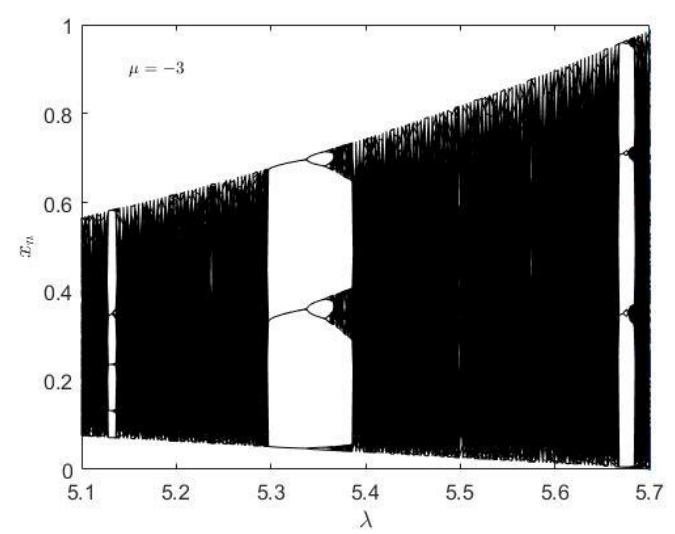

Figure 8. Period-3 window for $\mu=-3$

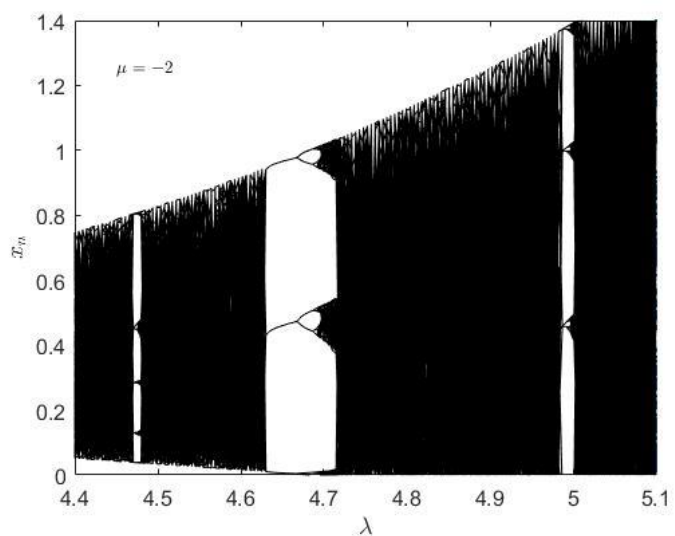

Figure 7. Period-3 window for $\mu=-2$

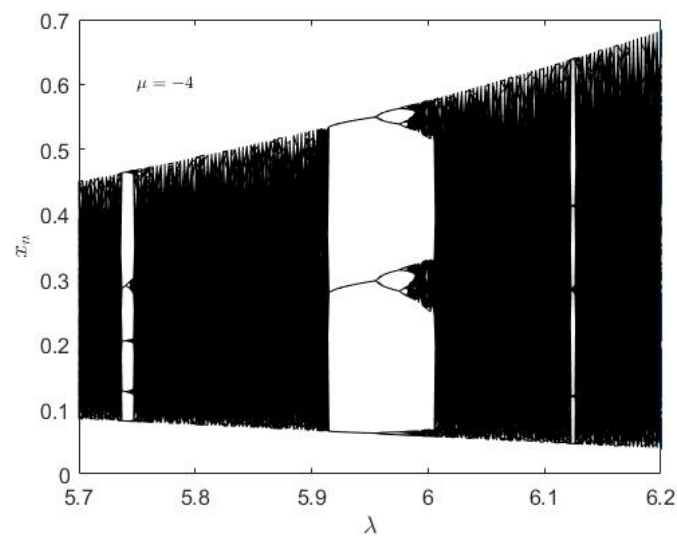

Figure 9. Period-3 window for $\mu=-4$

The Lyapunov exponents are presented in the next section corresponding to values of parameters $\lambda$ and $\mu$ from the bifurcation diagrams for which period-doubling occurs.

\section{Lyapunov Exponents and Chaotic Behaviour}

The chaotic behaviour in the family of functions considered is explored by using Lyapunov exponents, i.e., to quantify the chaos we see the nature of divergence of the nearby trajectories. In other words, to observe the chaos in the dynamics, we have to see the sensitive dependence on the initial conditions. To do this, we compute iterations of $g_{\lambda, \mu} \in G$ and determine trajectories of two nearby initial values $x_{0}=0.92$ and $x_{0}^{\prime}=0.97$ for $\mu=-1$ and $\lambda=4.85$. From Figure 10 , it is easily found that after few iterations both trajectories are more separated. This shows the divergence of nearby trajectories in the dynamics of $g_{\lambda, \mu} \in G$ for $\mu=-1$ and $\lambda=4.85$. Such a behaviour is chaotic since trajectories are immensely sensitive to initial conditions. It is provided that the two trajectories are separated which shows the divergence of nearby trajectories. It is evident that 
International Journal of Mathematical, Engineering and Management Sciences

Vol. 5, No. 6, 1191-1209, 2020

https://doi.org/10.33889/IJMEMS.2020.5.6.090

Lyapunov exponents measure the rate of divergence of initially close trajectories. The separation distance of two trajectories $\left\{x_{i}\right\}$ and $\left\{x_{j}\right\}$ is defined as $\left|x_{i}-x_{j}\right|$.

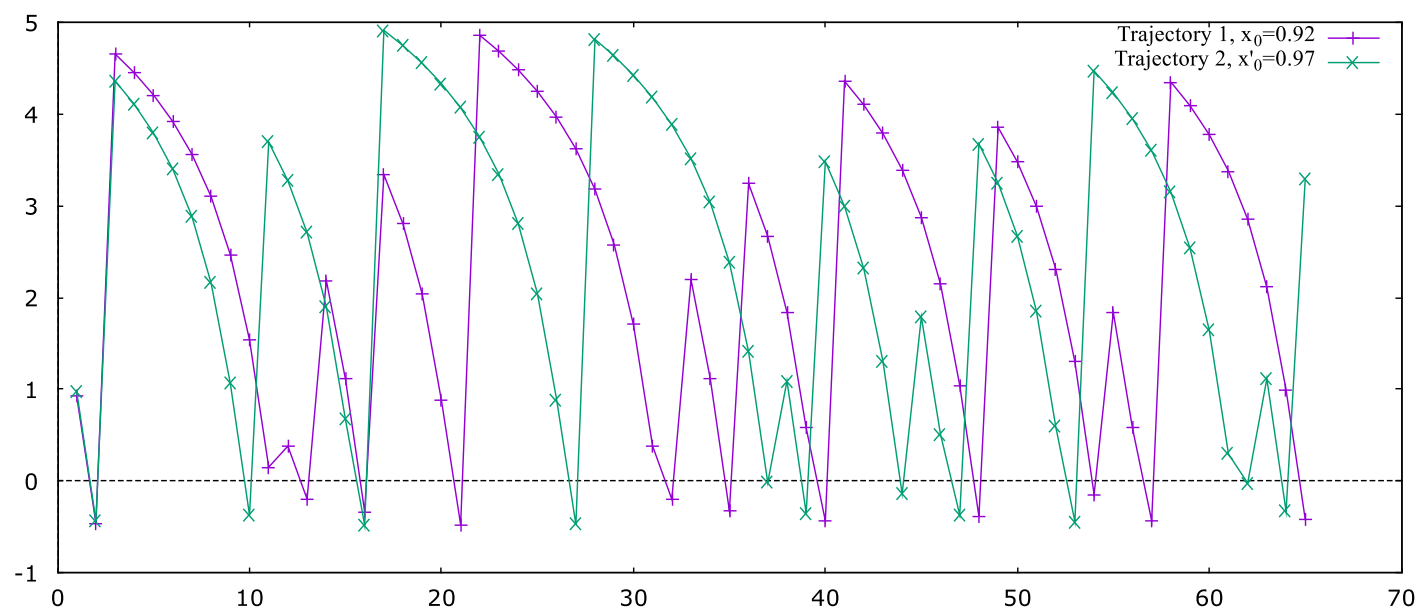

Figure 10. Two trajectories of two nearby initial values $x_{0}=0.92$ and $x_{0}^{\prime}=0.97$ for $\mu=-1$ and $\lambda=4.85$

The separation distance values between two trajectories $\{0.92,-0.45967,4.65428,4.44889$, $4.20831,3.91965,3.56219, \ldots . ., 0.99338,-0.42047\}$ and $\{0.97,-0.43431,4.36169,4.10459$, $3.79268,3.40070,2.88399, \ldots \ldots .,-0.33105,3.29703\}$ for $\mu=-1$ and $\lambda=4.85$ are computed and their magnitudes of difference $\{0.05,0.02535981,0.29259087,0.34430021,0.41563103$, $0.51895840,0.67819524, \ldots \ldots, 1.32442651,3.71750345\}$ are plotted in Figure 11.

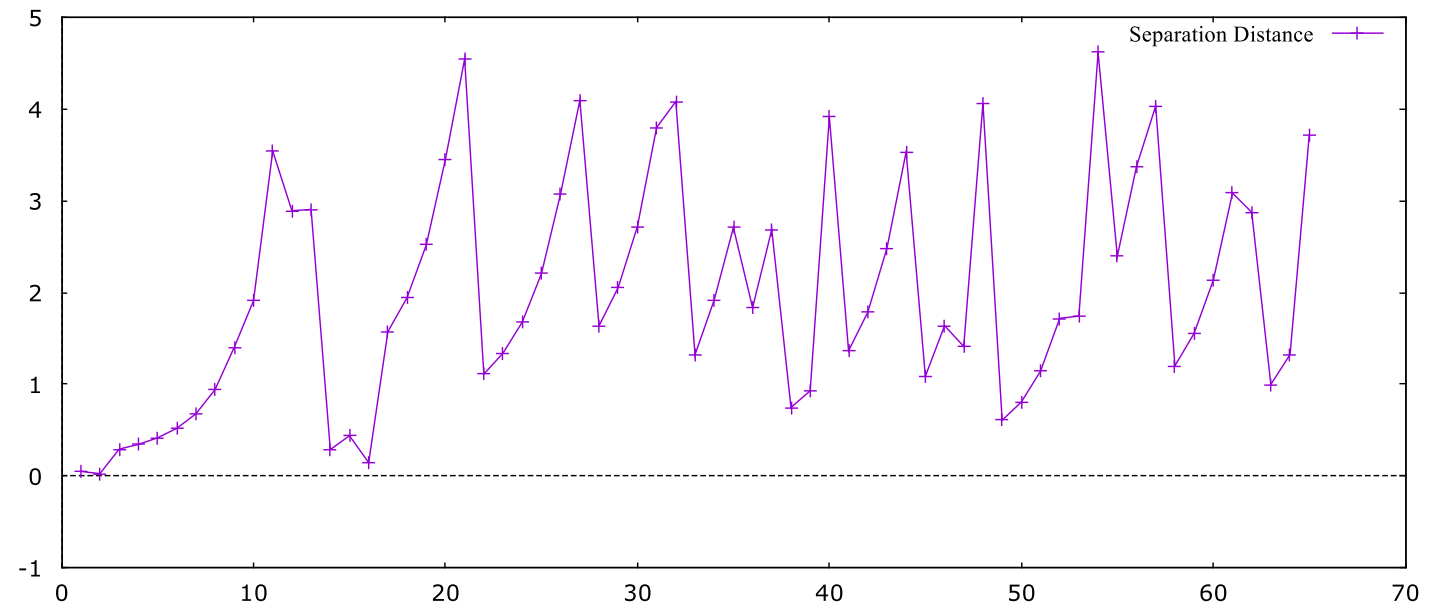

Figure 11. Separation distance trajectories starting with $x_{0}=0.92$ and $x_{0}^{\prime}=0.97$ for $\mu=-1$ and $\lambda=4.85$ 
International Journal of Mathematical, Engineering and Management Sciences

Vol. 5, No. 6, 1191-1209, 2020

https://doi.org/10.33889/IJMEMS.2020.5.6.090

Since the separation distance between two trajectories varies irregularly; it indicates the sensitive dependence on initial conditions. We observe that the separation distance values between the two trajectories cannot diverge continuously. The distance increases little fast for a while and after that distance decreases and increases, so we say that these trajectories $g_{\lambda, \mu} \in G$ starting from $x_{0}=0.92$ and $x_{0}^{\prime}=0.97$ diverge for selected parameters $\mu=-1$ and $\lambda=4.85$. To see the nature of divergence of the nearby trajectories for some ranges of parameter $\lambda$ for some fix $\mu$, the the Lyapunov exponent of $g_{\lambda, \mu} \in G$ is simulated by applying Formula (1)

$$
L=\lim _{j \rightarrow \infty} \frac{1}{j} \sum_{i=0}^{j-1} \ln \left|1+\left\{\mu-\lambda\left(1+\mu x_{i}\right)\right\} e^{\mu x_{i}}\right| .
$$

We pick $j=5000$ and $x_{0}=0.5$ in the computation. The calculated values of Lyapunov exponents are plotted in Figures $12,13,14$ and 15 for $\mu=-1,-2,-3,-4$ with $0.5 \leq \lambda \leq 7$, $0.5 \leq \lambda \leq 7.5,0.5 \leq \lambda \leq 8$ and $0.5 \leq \lambda \leq 8.5$ respectively.

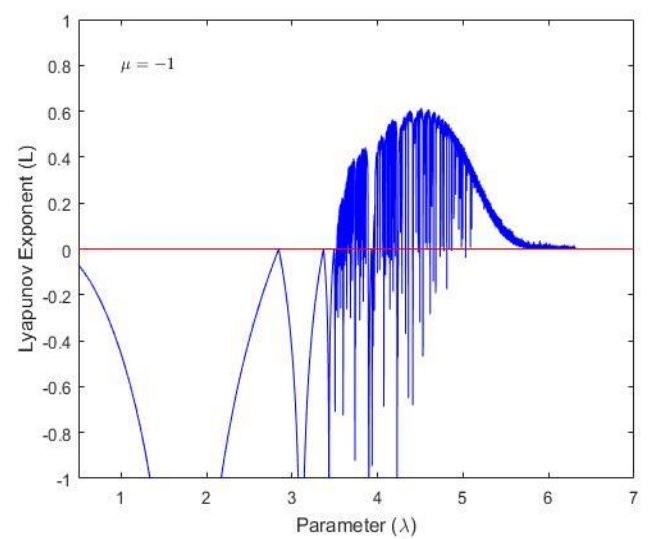

Figure 12. Lyapunov exponents for $\mu=-1$

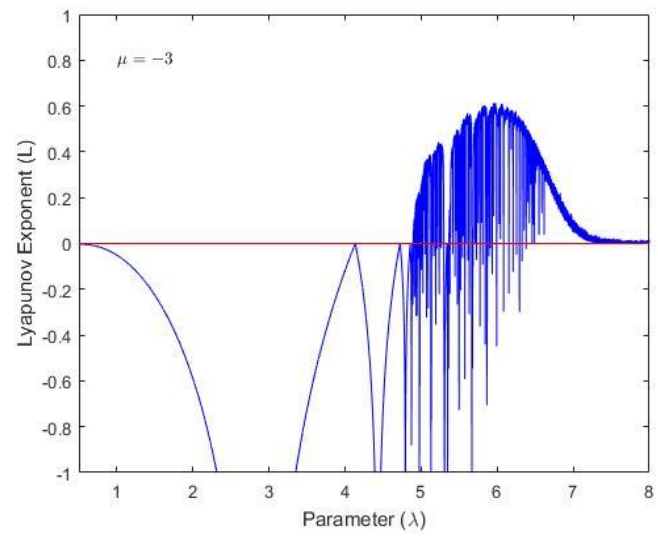

Figure 14. Lyapunov exponents for $\mu=-3$

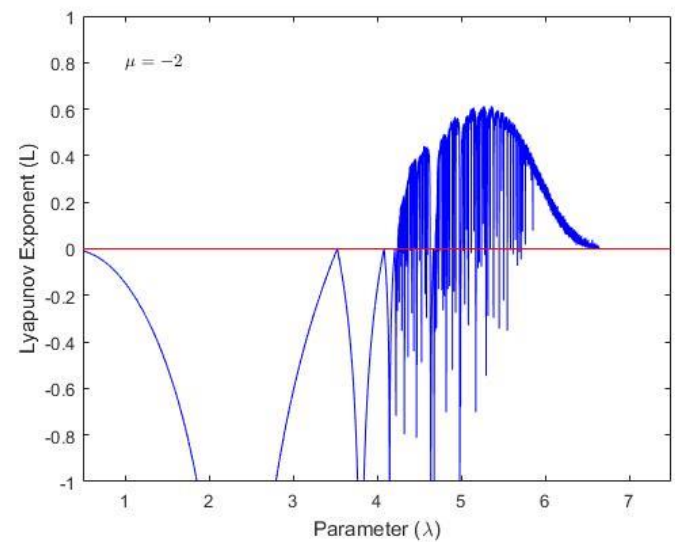

Figure 13. Lyapunov exponents for $\mu=-2$

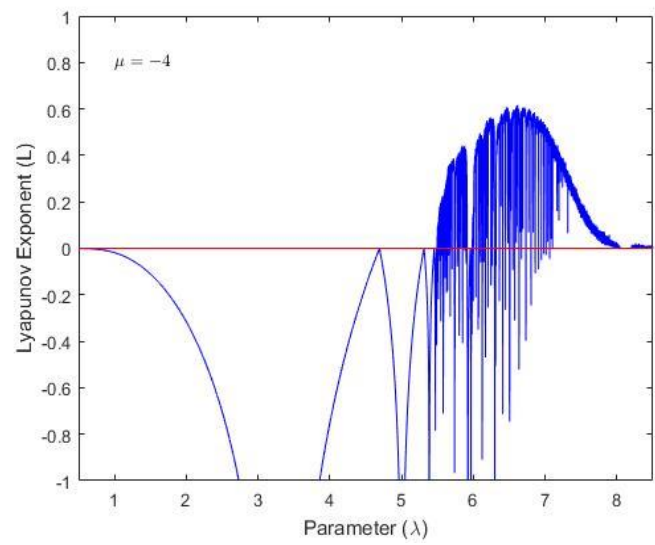

Figure 15. Lyapunov exponents for $\mu=-4$ 
International Journal of Mathematical, Engineering and Management Sciences

Vol. 5, No. 6, 1191-1209, 2020

https://doi.org/10.33889/IJMEMS.2020.5.6.090

For certain ranges of parameter $\lambda$, it is seen from the above figures that the Lyapunov exponents are positive. This shows that there is chaos present in the real dynamics of $g_{\lambda, \mu} \in G$. For $\mu=-1,-2,-3,-4$ and certain intervals of $\lambda$, the dark regions in bifurcation diagrams (Figures 2, 3, 4 and 5) corresponding to positive Lyapunov exponents (Figures 12,13,14 and 15) represent the chaos in the real dynamics $g_{\lambda, \mu}(x)$. Further, for some ranges, the bifurcation diagrams have white regions corresponding to negative values of Lyapunov exponents which expresses the nonchaotic region coming up temporarily.

\section{Conclusion}

In this article, chaos in transcendental functions from the two-parameter family associated with exponential map have been mainly studied. The real fixed points with their nature have been found theoretically although the periodic points have been simulated numerically. By period-doubling as well as by period-three window in bifurcation diagrams, the existence of chaos has been explored. Chaos is recognized by calculating positive Lyapunov exponents. As the possibility of further research in this direction, such type of results may be extended to functions having (i) 3 or more parameters, (ii) dimension 2 or higher.

\section{Conflict of Interest}

The authors declare that there is no conflict of interest for this research work.

\section{Acknowledgments}

The authors gratefully acknowledge Deanship of Scientific Research, Qassim University, Saudi Arabia for providing financial support to complete this research work under the project grant no.3508-qec-2018-1-14-S during 20182019AD/1440-1441AH.

\section{References}

Agliari, A., Naimzada, A., \& Pecora, N. (2017). Nonlinear monetary policy rules in a pure exchange overlapping generations model. Journal of Evolutionary Economics, 27, 1181-1203.

Banerjee, S., Rondoni, L., \& Mitra, M. (2013). Applications of chaos and nonlinear dynamics in science and engineering. Springer Berlin, Heidelberg.

Belyaev, A., \& Ryazanova, T. (2019). Stochastic sensitivity of attractors for a piecewise smooth neuron model. Journal of Difference Equations and Applications, 25(9-10), 1468-1487.

Brown, R.J. (2018). A modern introduction to dynamical systems. Oxford University Press, New York.

Chakra, T.K., \& Nayak, T. (2019). Iteration of the translated tangent. Bulletin of the Malaysian Mathematical Sciences Society, 42(5), 1993-2008.

de Carvalho, R.E., \& Leonel, E.D. (2016). Squared sine logistic map. Physica A: Statistical Mechanics and its Applications, 463, 37-44.

Devaney, R.L. (2001). Sex : dynamics, topology, and bifurcations of complex exponentials. Topology and its Applications, 110(2), 133-161.

Gritli, H., \& Belghith, S. (2015). Computation of the Lyapunov exponents in the compass-gait model under OGY control via a hybrid Poincaré map. Chaos, Solitons \& Fractals, 81, 172-183. 
International Journal of Mathematical, Engineering and Management Sciences

Vol. 5, No. 6, 1191-1209, 2020

https://doi.org/10.33889/IJMEMS.2020.5.6.090

Gritli, H., \& Belghith, S. (2017). Walking dynamics of the passive compass-gait model under OGY-based control: emergence of bifurcations and chaos. Communications in Nonlinear Science and Numerical Simulation, 47, 308-327.

Gulick, D. (2012). Encounters with chaos and fractals. CPC Press, New York.

Kapoor, G.P., \& Prasad, M.G.P. (1998). Dynamics of $\left(\mathrm{e}^{\wedge}\{\mathrm{z}\}-1\right) / \mathrm{z}$ : the Julia set and bifurcation. Ergodic Theory and Dynamical Systems, 18(6), 1363-1383.

Lakshmanan, M., \& Rajasekar, S. (2003). Nonlinear dynamics: integrability, chaos, and patterns. SpringerVerlag Berlin, Heidelberg.

Letellier, C. (2019). Chaos in nature. World Scientific Series on Nonlinear Science Series A, $2^{\text {nd }}$ Edition, Singapore.

Lim, D. (2016). Fixed points and dynamics on generating function of Genocchi numbers. Journal of Nonlinear Sciences and Applications, 9(3), 933-939.

Liu, L., \& Miao, S. (2018). A new simple one-dimensional chaotic map and its application for image encryption. Multimedia Tools and Applications, 77, 21445-21462.

Magreñán, Á.A. (2014). A new tool to study real dynamics: the convergence plane. Applied Mathematics and Computation, 248, 215-224.

Mickens, R.E. (2015). Difference equations: theory, applications and advanced topics. CRC Press, Taylor \& Francis Group, Boca Raton.

Nayak, T., \& Prasad, M.G.P. (2014). Julia sets of Joukowski-exponential maps. Complex Analysis and Operator Theory, 8(5), 1061-1076.

Özer, M., Čenys, A., Polatoglu, Y., Hacibekiroglu, G., Akat, E., Valaristos, A., \& Anagnostopoulos, A.N. (2007). Bifurcations of Fibonacci generating functions. Chaos, Solitons \& Fractals, 33(4), 1240-1247.

Radwan, A.G. (2013). On some generalized discrete logistic maps. Journal of Advanced Research, 4(2), 163171.

Sajid, M. (2015a). On real fixed points of one parameter family of function $\mathrm{x} /\left(\mathrm{b}^{\wedge}\{\mathrm{x}\}-1\right)$. Tamkang Journal of Mathematics, 46(1), 61-65.

Sajid, M. (2015b). Singular values and fixed points of family of generating function of Bernoulli's numbers. Journal of Nonlinear Sciences and Applications, 8(1), 17-22.

Sajid, M. (2016). Real fixed points and dynamics of one parameter family of function $\left(b^{x}-1\right) / x$. Journal of the Association of Arab Universities for Basic and Applied Sciences, 21(1), 92-95.

Sajid, M. (2018). Bifurcation and chaos in real dynamics of a two-parameter family arising from generating function of generalized Apostol-type polynomials. Mathematical and Computational Applications, 23(1), 7.

Sajid, M. (2019). Chaotic behavior in real dynamics and singular values of family of generalized generating function of Apostol-Genocchi numbers. Journal of Mathematics and Computer Science, 19(1), 41-50.

Sajid, M., \& Kapoor, G.P. (2007). Dynamics of transcendental meromorphic functions $\frac{z+\mu_{0}}{z+\mu_{0}+4} e^{z}$ having rational Schwarzian derivative. Dynamical Systems.: An International Journal, 22(3), 323-337.

Sharifi, S., Salimi, M., Siegmund, S., \& Lotfi, T. (2016). A new class of optimal four-point methods with convergence order 16 for solving nonlinear equations. Mathematics and Computers in Simulation, 119, $69-90$. 
International Journal of Mathematical, Engineering and Management Sciences

Vol. 5, No. 6, 1191-1209, 2020

https://doi.org/10.33889/IJMEMS.2020.5.6.090

Skiadas, C.H., \& Skiadas, C. (2016). Handbook of applications of chaos theory. Chapman and Hall/CRC. New York.

Stavroulaki, M., \& Sotiropoulos, D. (2012). The energy of generalized logistic maps at full chaos. Chaotic Modeling and Simulation, 3, 543-550.

Thompson, J.M.T. (2016). Chaos, fractals and their applications. International Journal of Bifurcation and Chaos, 26(13), 1630035. 